\title{
Off the Shortest Path: Betweenness on Street Network Level to Study Pedestrian Movement
}

\author{
Stephen Law \\ Alan Turing Institute \\ British Library, 96 Euston Road, London NW1 2DB \\ London, UK \\ Email: slaw@turing.ac.uk
}

\author{
Martin Traunmueller \\ Center for Urban Science \& Progress \\ New York University \\ Brooklyn, NY 11201 \\ Email: martin.traunmueller@nyu.edu
}

\begin{abstract}
Betweenness centrality is an important measure in network sciences that reflects the extent a node lies in between any pairs in a graph. The measure has been used by urban studies, to discuss the relationship between urban mobility and the spatial street network of a city, using Dijkstra shortest path betweenness centrality to describe human wayfinding procedures. As in reality, wayfinding is a more complex endeavor, results of studies using both random path or the most optimal shortest path approach might be misleading.

In this paper we propose with the exploratory betweenness centrality (EBC) a more realistic set of measures that uses an exploratory path in calculating centrality rather than an optimal path in studying pedestrian movement. In particular we calculate EBC where the agent selects the longest street nearest to the destination (App-EBC) or any random street that is approaching the destination (Ran-EBC)

In doing so, we compare how EBC and GBC correlate with aggregate pedestrian movement for two case studies in London. The result shows the EBC measures explains equal or greater variation of aggregate pedestrian movement than the GBC measure for both of the case studies, indicating the potential of using measures of EBC in modeling urban mobility.

keywords - Street networks, space syntax, wayfinding, shortest
\end{abstract} path, spatial cognition, betweenness centrality, urban mobility

\section{INTRODUCTION}

Betweenness centrality (BC) [1], measures for a particular node the number of overlaps between every pair of origin and destination in a graph. The descriptive measure has been important for identifying the person that is between many others in a social network or the node that has the greatest risk for congestion in a communication network. Applied on the street network, the centrality measure found strong correspondence with aggregate pedestrian movement distribution [2]. Despite its descriptive importance, limited research in pedestrian navigation and movement analysis has been done on the shortest path approach most work grounds its findings on. One common algorithm for solving the shortest path problem is the Dijkstra [3] algorithm which finds in a weighted graph the shortest path between any pair of origin and destination assuming global knowledge of the system. Recent research have also suggested the use of random walk in calculating betweenness through a markov chain process[4]. However this type of measures is also unrealistic in the majority of wayfinding activities. Pedestrians might not have complete knowledge nor zero knowledge of the system when navigating but rather partial knowledge, hence where the shortest path is limited. To verify this, a simple correlation was conducted between pedestrian movement and current flow betweenness [4] for one of the case studies in London. As expected, the result shows a poor association between the two which suggest that pedestrian movement is closer to optimal and further than random.

Inspired from spatial cognition and space syntax research, we propose the exploratory betweeness centrality measure offering statistically stronger results, which takes an exploratory path rather than an optimal path when calculating betweenness centrality. This paper is structured as follows: First, we outline previous research on pedestrian navigation, including a description of the exploratory betweenness centrality (EBC) and the global betweenness centrality (GBC) measure. Then, we describe our empirical method to explore the difference through two case studies in London. Last, we present our results and discuss limitations and opportunities.

\section{BACKGROUND}

\section{A. Literature}

Wayfinding [5][6] involves a complex cognitive process for identifying the paths between origins and destinations that are influenced by its spatial configuration, signage, built environment and visual access [7].

In this work, we focus on the aspect of spatial network configuration from the field of space syntax into applying the graph perspective on street networks into understanding pedestrian movement and navigation [8]. From the spatial network perspective, two general strategies are often employed. One strategy is to take the least-effort metric shortest path to reach its destination and the other strategy is to take the simplest path such as minimizing the number of turns or preserving linearity to reaching its destination [9][2]. In reality wayfinding is a complex process and sits somewhere between the two, as pedestrians, not familiar to an urban area, are more likely to take a least turn path using less information while people more familiar with an area are likely to use a more complex strategy, such as backroads.

In addition, previous work also shows that pedestrians are not necessarily taking an optimal path [10] but actually are in a constant process of routes evaluation based on both 
the local information, their bearings to the destination and the user knowledge of the system. These findings have been supported by research in spatial cognition such as our homing instinct in giving us a natural sense of direction [11]. We are also constantly retrieving topological information from our hippocampus informing these wayfinding decisions [12] [13] [14]. Furthermore, outcome can also be related to the concept of bounded rationality [15] in behavioural economics where users might not have full information to make a utilitymaximised decision. From this evidence, the use of an optimal path in calculating betweenness centrality to study pedestrian movement can therefore be unrealistic.

Recent pedestrian wayfinding research generally supported this sub-optimal strategy that includes looking at anchor-based strategies [16] and hierarchical strategy [17]. A recent study from the author [18] began to compare the difference between a suboptimal path and an optimal shortest path. The result shows the two matches approximately $70-80 \%$ of the time. Inspired from spatial cognition and space syntax research, this research propose the exploratory path betweenness centrality in studying aggregate pedestrian movement.

\section{B. Betweenness Centrality}

The idea of centrality was first applied to social network by Bevelas [19]. Since then, various centrality measures had been proposed including degree, closeness, betweenness centrality [20]. In the field of space syntax, angular betwenness (BC) of the street network have been found to correlate significantly with aggregate pedestrian movement [2]. For a weighted graph $\mathrm{G}=(\mathrm{V}, \mathrm{E})$ made up of nodes $(\mathrm{V})$ and edges $(\mathrm{E})$. The set $\mathrm{V}$ represent the streets and the set $\mathrm{E}$ represent the junctions. $\mathrm{BC}$ measures the number of shortest paths overlap on street segment (v) from all origin and destination pairs in a graph. Higher BC indicates a greater probability a street segment is traversed or passed through. More formally, BC can be described as:

$$
B C(v)=\sum_{s \neq t} \theta_{s t}(v) / \theta_{s t}
$$

where $\theta_{s t}$ denotes all the shortest path from $\mathrm{s} \in \mathrm{V}$ and $\mathrm{t} \in$ $\mathrm{V}$ and $\theta_{s t}(\mathrm{v})$ denotes the shortest path from $\mathrm{s}$ and $\mathrm{t}$ that lies on $\mathrm{v} \in \mathrm{V}$. [1] BC is furthermore differentiated in Global and Exploratory Betweenness Centrality.

Global Betweenness Centrality: Brandes [1] proposed the use of the Dijkstra shortest path algorithm in calculating BC for a weighted graph. Adapted from the Breadth-first-search (BFS) algorithm, the Dijkstra shortest path algorithm [3] starts from the origin and it searches every neighbours and then every neighbor's-neighbors recursively until it reaches the destination. A shortest path is then identified by minimising the global shortest path length between the origin and destination. This research calls the Brandes algorithm here the Global Betweenness Centrality (GBC) as it finds a global optimal path. This can be applied on any weighted graph. Following previous pedestrian movement research [2], we use both the

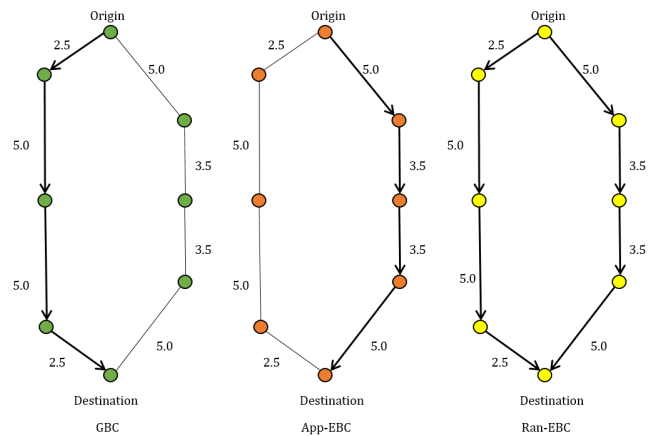

Fig. 1. Diagram highlighting the path selected by GBC on the right, App-EBC in the centre and Ran-EBC on the right for an abstract graph.

metric and angular costs between streets as edge weights in calculating the GBC measure.

Exploratory Betweenness Centrality: Exploratory Betweenness Centrality (EBC) is here defined as a set of measures that uses an exploratory path in calculating centrality. A key characteristic of the exploratory path is it does not back-track. In another words, there is a single path between $\mathrm{s}$ and $\mathrm{t}$ where an agent trims the path tree after making a decision even if it is not necessarily the shortest. The denominator of EBC is therefore set to 1 . This can simply be a random-walk path, a locally optimal path or simply a path that approaches the destination. This research uses the latter in calculating EBC for the study where the agent has the knowledge if it is closer to the destination or not while walking. This is as oppose to a pure random walk.

We call the first variation APP-EBC where the exploratory agent at each step is selecting the longest street nearest to the destination. This is repeated at every node until it reaches the destination. This strategy is based on the intuition that the pedestrian is afforded limited visual information and that taking the longest street can possibly bring you closer to the destination at the next wayfinding-point. The second variation is Ran-EBC where the exploratory agent is picking randomly any street that is approaching the destination. This is repeated at every node until it reaches the destination. This strategy is based on the intuition that pedestrians do not have a strong preference in path selection as long as it is homing towards the destination. We use both the metric and angular distance between streets as edge weights in calculating both EBC measures. Figure 1 shows an abstract example to demonstrate the difference between GBC, App-EBC and RanEBC where the aim is to find a path from node "Origin" to node "Destination" according to the edge weights. The picture on the left shows the shortest path taken by the GBC measure. The picture in the middle shows the path taken by the APPEBC measure. The picture on the right shows both paths as either paths could be selected at random by the Ran-EBC measure. 


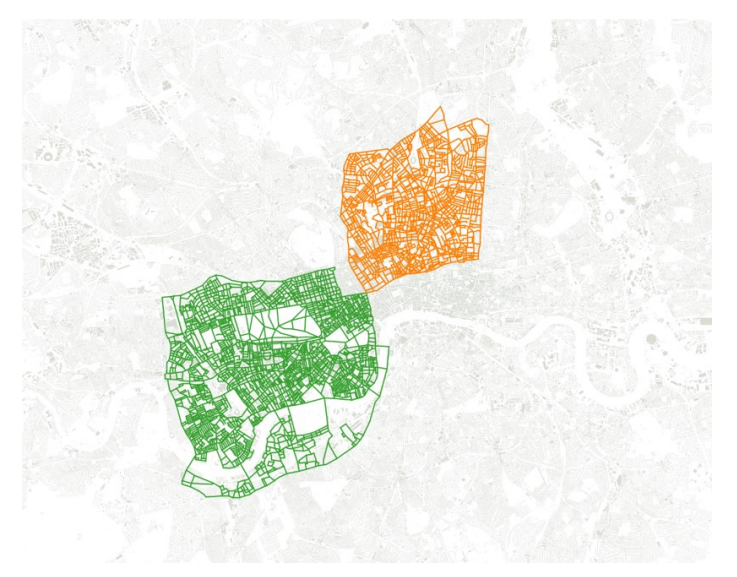

Fig. 2. Map of Barnsbury and Kensington. Contains Ordnance Survey data Crown copyright and database right [2016]

\section{CASE Study ANd Datasets}

We follow the general empirical procedure from Penn [21] and Hillier and Iida [2] in correlating aggregating pedestrian movement and street network centrality. We select two of the case studies from the dataset namely Barnsbury and Kensington in London. Barnsbury is a predominately residential area to the north of London and Kensington is a retail, mixed-use residential area in West London. Figure 2 shows the two case study areas where the orange lines indicate the street network for the Barnsbury study area and the green lines indicate the street network for the Kensington study area. To study the correlation between centrality and movement patterns, we need information describing the road network and pedestrian movement. Next we will describe our two datasets at hand, providing this information.

\section{A. Street Network Dataset}

Our first dataset describing the street network is the Meridian Line Street network dataset, open source for London and publicly available through Ordinance Survey (OS) open data framework. (OS open data 2016) We have manually added the pedestrian paths and missing links for the empirical analysis. In order to calculate the $\mathrm{BC}$ measures on the street network, the dataset is converted into first a dual graph or a line graph where the node is the street and the edge is the junction following the standard procedure in space syntax research [2]. We then calculate two versions of the App-EBC, Ran-EBC and GBC algorithm for each of the case study. The first uses metric distance as edge weights. The second uses the angularity as edge weights. This results in six measures being calculated for the two case studies.

Figure 3 shows the histogram of the six $\mathrm{BC}$ measures for the Barnsbury study area. The histogram shows all six measures of betweenness centrality are rightly-skewed suggesting that there are many low betweenness streets and fewer high betweenness streets. Figure 4 shows in a GIS mapping the spatial distribution for the six BC measures in Barnsbury as a heatmap where red denotes higher betweenness centrality and
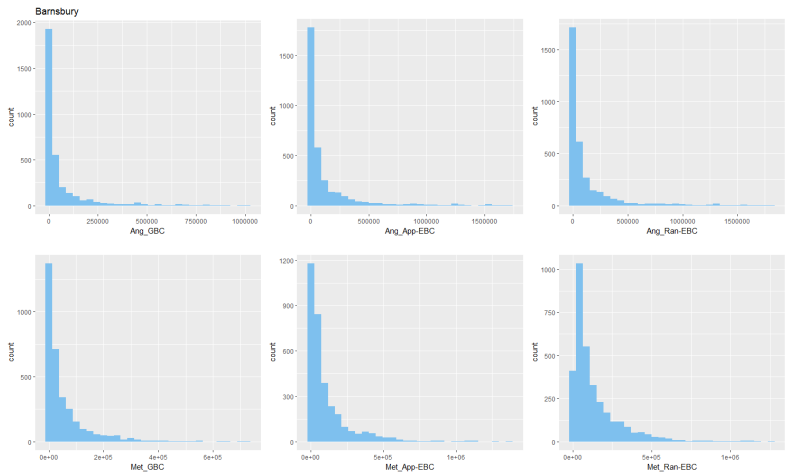

Fig. 3. histogram of Barnsbury BC
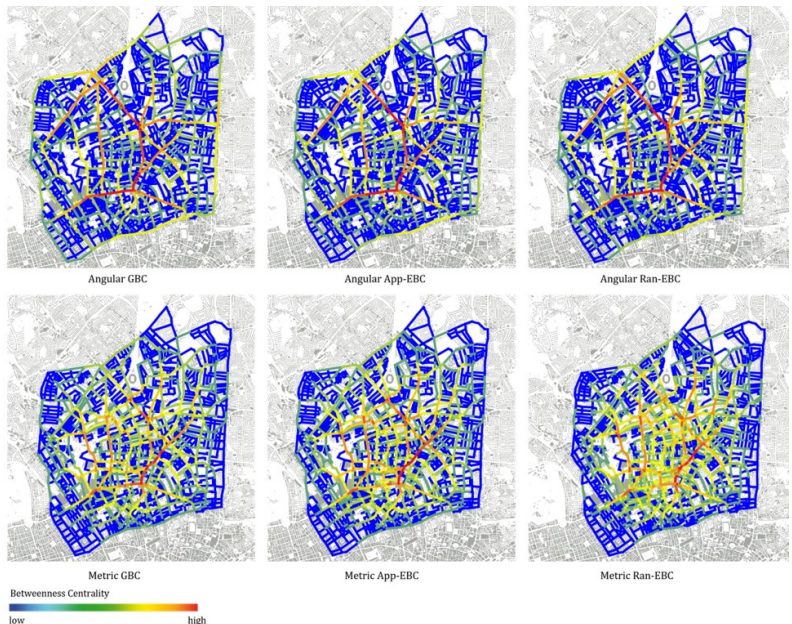

Fig. 4. Betweenness centrality of the Barnsbury Street network. Contains Ordnance Survey data Crown copyright and database right [2016]

blue denotes lower betweenness centrality. The visualisation shows Betweenness centrality captures the structure of the street network system where the main routes are highlighted in red and the least used routes highlighted in blue. Despite the differences in individual paths, the result shows GBC and EBC are visually similar. This is expected on aggregate as the path which approaches the destination one step at a time will also likely use the main routes in reaching the destination. The angular weight measures show greater concentration on the main streets while the metric weights measure is clearly more distributed where the back roads are also highlighted. Due to similar observations, histograms and visualisations are not repeated in Kensington for brevity reasons.

\section{B. Pedestrian Movement Dataset}

The second is the pedestrian movement dataset collected from a previous study [21]. The pedestrian movement dataset contains the average hourly pedestrian movement for a particular street segment. Figure 5 is a histogram showing the pedestrian movement distribution for the Barnsbury area on the left and the Kensington area on the right. The pedestrian movement distribution is rightly-skewed which suggests that 


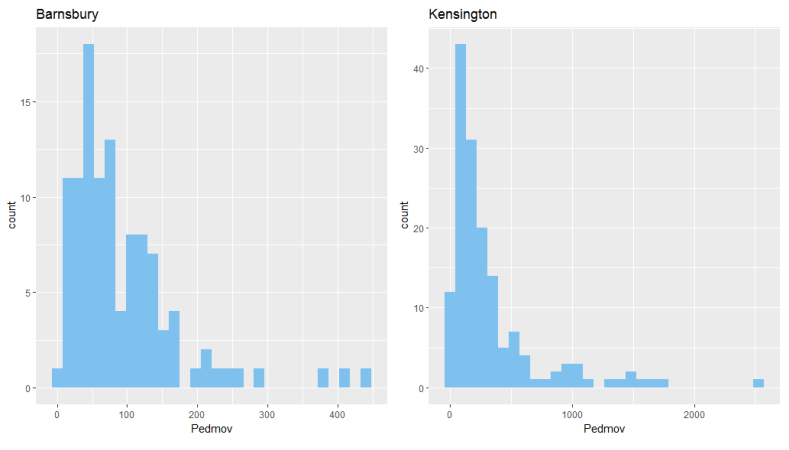

Fig. 5. Pedestrian movement distribution for Barnsbury and Kensington

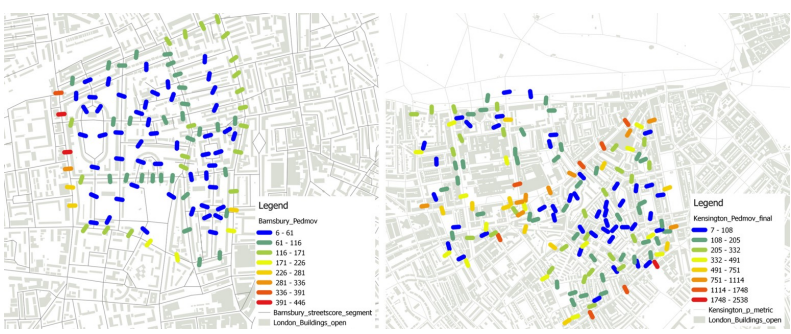

Fig. 6. Pedestrian movement mapped for Barnsbury and Kensington

there are many low pedestrian movement streets and few high pedestrian movement streets. Figure 6 maps the pedestrian movement for the Barnsbury area on the left and the Kensington area on the right respectively. Pedestrian movement is visualized in a heatmap where red denotes higher pedestrian movement and blue denotes lower pedestrian movement. As can be seen from the two figures, there are high pedestrian flows along the straightest and most continous routes such as Knightsbridge and Brompton Road in the Kensington area and Caledonian Road in the Barnsbury area. While the other streets are much quieter. This corresponds visually to the betweenness centrality. The result also shows the Kensington area has generally a higher and more distributed pedestrian flow pattern that is influenced by both the transport attraction such as the London Underground station and the urban attraction such as the various museums in the area. Barnsbury on the other hand is a more residential area with a line of shops along Caledonian Road.

\section{Methods}

The aim of the study is to empirically test to what extent GBC, App-EBC and Ran-EBC differ when correlating with aggregate pedestrian movement. To do so we run an ordinary least square (OLS) linear regression model between the log of the aggregate pedestrian movement and the $\log$ of the betweenness centrality measures, as shown in Equation (3). This is repeated for GBC, App-EBC and Ran-EBC. For each model both angular weights and metric weights are tested. This results in six regression models. Basic statistics such as goodness of fit (R2) and P-value are reported.

$$
\log \left(\operatorname{Pedmov}_{i}\right)=\beta_{1} \log \left(B C_{i}\right)+\epsilon_{i}
$$

Let Pedmov to denote the hourly pedestrian movement of segment (i) and $\mathrm{BC}$ to denote the betweenness centrality of segment (i).

\section{RESULTS}

Table I and table II shows the descriptive statistics for the empirical analysis. This includes for each street, the pedestrian flow, the angular and metric GBC, App-EBC and Ran-EBC.

\begin{tabular}{lccccc}
\hline \hline Statistic & N & Mean & St. Dev. & Min & Max \\
\hline id & 109 & 55.0 & 31.6 & 1 & 109 \\
Pedmov & 109 & 92.1 & 79.1 & 6 & 446 \\
Ang_GBC & 109 & $73,414.6$ & $105,649.8$ & 1 & 524,041 \\
Met_GBC & 109 & $87,007.5$ & $86,637.1$ & 748 & 322,100 \\
AngApp_EBC & 109 & $153,524.5$ & $199,237.5$ & 3,321 & 876,776 \\
MetApp_EBC & 109 & $172,407.9$ & $163,862.6$ & 9,337 & 670,136 \\
AngRan_EBC & 109 & $161,319.9$ & $204,269.0$ & 3,559 & 898,613 \\
MetRan_EBC & 109 & $212,144.0$ & $137,139.8$ & 8,929 & 604,559 \\
\hline \multicolumn{7}{c}{ TABLE I } \\
& BARNSBURY DESCRIPTIVE STATISTICS
\end{tabular}

\begin{tabular}{lccccc}
\hline \hline Statistic & $\mathrm{N}$ & Mean & St. Dev. & Min & Max \\
\hline id & 155 & 78.1 & 45.0 & 1 & 156 \\
Pedmov & 155 & 331.0 & 399.8 & 7 & 2,538 \\
Ang_GBC & 155 & $201,212.0$ & $364,981.1$ & 0 & $1,854,038$ \\
Met_GBC & 155 & $197,574.4$ & $253,018.8$ & 91 & $1,069,253$ \\
AngApp_EBC & 155 & $403,430.9$ & $705,387.2$ & 5,528 & $3,806,522$ \\
MetApp_EBC & 155 & $409,635.9$ & $522,723.3$ & 9,519 & $2,562,309$ \\
AngRan_EBC & 155 & $423,541.3$ & $733,362.0$ & 5,528 & $3,960,129$ \\
MetRan_EBC & 155 & $498,811.6$ & $457,762.9$ & 30,563 & $2,336,595$ \\
\hline
\end{tabular}

KENSINGTON DESCRIPTIVE STATISTICS

Figure 7 shows the log-log scatterplot between pedestrian movement and betweenness centrality for the Barnsbury area and Table III shows the regression results. In accordance to the F-statistics, all six models are significant at the P-value 0.01 level and the R2 is between $58-74 \%$ for the Barnsbury area. The result shows in all six models betweenness centrality can explain the majority of the pedestrian movement variation using strictly the street network properties. The R2 for AppEBC improves by $6-12 \%$ in comparison to GBC. The R2 for Ran-EBC improves by $12 \%$ using angular weights but reduce by $11 \%$ using metric weights in comparison to GBC. In general EBC measures have stronger fit to the data than GBC where Ang-Ran-EBC achieves the highest R2.

Figure 8 shows the log-log scatterplot between pedestrian movement and betweenness centrality for the Kensington area and table IV shows the corresponding regression results. In accordance to the F-statistics, all four models are significant at the P-value 0.01 level and the R2 is $48-55 \%$ for the Kensington area and hence, can explain the majority of the pedestrian movement variation. The $\mathrm{R} 2$ for App-EBC improves by $2-8 \%$ and the R2 for Ran-EBC improves by 1-3\% in comparison to GBC. Similarly, this shows clearly EBC measures have stronger fit to the data than GBC measures where Ang-RanEBC achieves the highest R2. 

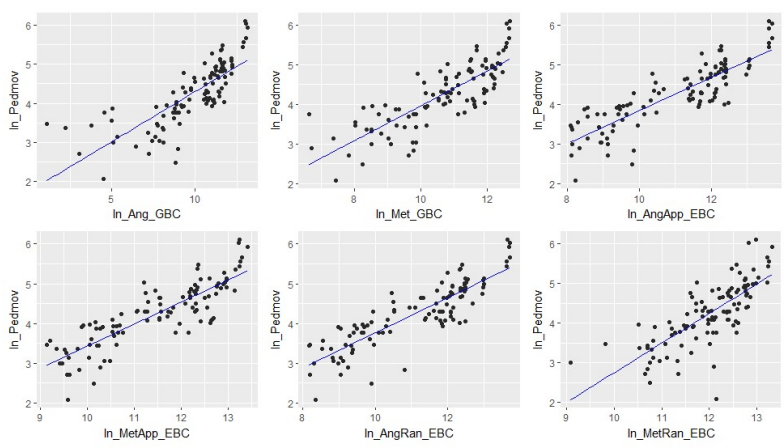

Fig. 7. Log-Log plot between pedestrian movement and BC for Barnsbury

\begin{tabular}{lcccccc}
\hline \hline & \multicolumn{5}{c}{ Dependent variable: } \\
\cline { 2 - 6 } & $(1)$ & $(2)$ & $(3)$ & Beta Estimates & $(4)$ & $(6)$ \\
\hline Ang_GBC & $0.255^{* * *}$ & & & & \\
& $(0.019)$ & & & & \\
Met_GBC & & $0.438^{* * *}$ & & & & \\
& & $(0.030)$ & & & &
\end{tabular}

\begin{tabular}{|c|c|c|c|c|c|c|}
\hline AngApp_EBC & & & $\begin{array}{c}0.418^{* * *} \\
(0.024)\end{array}$ & & & \\
\hline MetApp_EBC & & & & $\begin{array}{c}0.556^{* * *} \\
(0.034)\end{array}$ & & \\
\hline AngRan_EBC & & & & & $\begin{array}{c}0.443^{* * * *} \\
(0.025)\end{array}$ & \\
\hline MetRan_EBC & & & & & & $\begin{array}{c}0.750^{* * * *} \\
(0.062)\end{array}$ \\
\hline Constant & $\begin{array}{c}1.747^{* * *} \\
(0.196)\end{array}$ & $\begin{array}{l}-0.417 \\
(0.327)\end{array}$ & $\begin{array}{l}-0.337 \\
(0.269)\end{array}$ & $\begin{array}{c}-2.134^{* * *} \\
(0.392)\end{array}$ & $\begin{array}{c}-0.666^{* *} \\
(0.283)\end{array}$ & $\begin{array}{c}-4.757^{* * *} \\
(0.751)\end{array}$ \\
\hline$\overline{\mathrm{Obs}}$ & 109 & 109 & 109 & 109 & 109 & 109 \\
\hline $\mathrm{R}^{2}$ & 0.617 & 0.659 & 0.735 & 0.715 & 0.741 & 0.575 \\
\hline Adj $R^{2}$ & 0.613 & 0.656 & 0.732 & 0.712 & 0.739 & 0.571 \\
\hline F Stat $(\mathrm{df}=1 ; 107)$ & $172.243^{* * *}$ & $207.145^{* * *}$ & $296.496^{* * *}$ & $268.601^{* * *}$ & $306.771^{* * *}$ & $144.521^{* * *}$ \\
\hline
\end{tabular}

BARNSBURY REGRESSION RESULTS

In summary, we can see the potential of betweenness centrality using the street network information to explain pedestrian movement variation [2]. We can also see betweenness centrality was able to explain greater pedestrian movement variations for the Barnsbury area in comparison to the Kensington area. This is logical as Barnsbury is more dominated by natural movement while Kensington is more dominated by functional movement. Betweenness Centrality assumes a relatively homogeneous distribution of origins and destinations in the study area. Therefore differences in attraction density is not considered from a pure spatial network perspective.

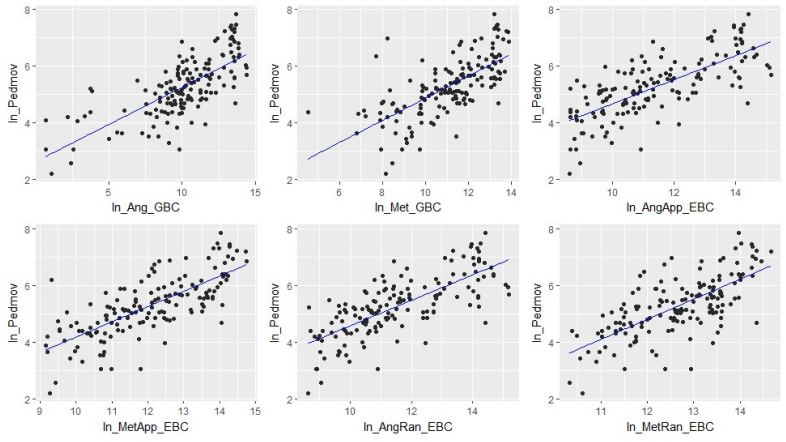

Fig. 8. Log-Log plot between pedestrian movement and $\mathrm{BC}$ for Kensington

\begin{tabular}{|c|c|c|c|c|c|c|}
\hline & \multicolumn{6}{|c|}{ Dependent variable: } \\
\hline & \multicolumn{6}{|c|}{ Beta Estimates } \\
\hline & (1) & (2) & (3) & (4) & (5) & (6) \\
\hline Ang_GBC & $\begin{array}{c}0.262^{* * *} \\
(0.020)\end{array}$ & & & & & \\
\hline Met_GBC & & $\begin{array}{c}0.391^{* * * *} \\
(0.033)\end{array}$ & & & & \\
\hline AngApp_EBC & & & $\begin{array}{c}0.424^{* * * *} \\
(0.032)\end{array}$ & & & \\
\hline MetApp_EBC & & & & $\begin{array}{c}0.538^{* * *} \\
(0.039)\end{array}$ & & \\
\hline AngRan_EBC & & & & & $\begin{array}{c}0.449^{* * *} \\
(0.033)\end{array}$ & \\
\hline MetRan_EBC & & & & & & $\begin{array}{c}0.706^{* * *} \\
(0.060)\end{array}$ \\
\hline Constant & $\begin{array}{c}2.631^{* * * *} \\
(0.215)\end{array}$ & $\begin{array}{l}0.956^{* *} \\
(0.373)\end{array}$ & $\begin{array}{c}0.431 \\
(0.371)\end{array}$ & $\begin{array}{c}-1.208^{* *} \\
(0.478)\end{array}$ & $\begin{array}{c}0.079 \\
(0.384)\end{array}$ & $\begin{array}{c}-3.670^{* * *} \\
(0.761)\end{array}$ \\
\hline Obs & 155 & 155 & 155 & 155 & 155 & 155 \\
\hline $\mathrm{R}^{2}$ & 0.517 & 0.474 & 0.532 & 0.550 & 0.550 & 0.476 \\
\hline Adj $R^{2}$ & 0.514 & 0.470 & 0.529 & 0.547 & 0.547 & 0.473 \\
\hline F Stat & $163.915^{* * *}$ & $137.759^{* * *}$ & $174.228^{* * *}$ & $186.725^{* * *}$ & $187.107^{* * *}$ & $139.071^{* * *}$ \\
\hline
\end{tabular}

Our findings also show that EBC explains generally greater pedestrian movement variation than GBC for both case studies in London, while Ang-Ran-EBC achieves the highest R2. This indicates that pedestrians might be taking a path that is approaching the destination but not necessarily the global optimal path.

\section{DISCUSSION}

In this paper we propose the exploratory betweenness centrality, a set of measures that takes an exploratory path rather than an optimal path when calculating betweenness in studying pedestrian movement. For the empirical study we tested App-EBC that selects the longest street nearest to the destination, Ran-EBC that selects any random street that approaches the destination and GBC that takes an optimal path to the destination in calculating different versions of betweenness centrality. We compare the three measures by correlating it with aggregate pedestrian movement for the two case studies in London. The result shows firstly that the network measures using street information can explain a great proportion of movement variation. The result also shows EBC explains statistically greater variation of aggregate 
pedestrian movement than GBC and are consistent for both case studies in London. Interpreting these initial results, there is a possibility we may be taking an exploratory path that is always heading closer (metric distance) or heading towards (angular distance) the destination when navigating but not necessarily the global optimal path at the aggregate level [10]. One interpretation is related to our homing instinct in navigating towards our destination [11]. Another interpretation is we might have partial knowledge [15] of the urban network as humans evaluate their decision tree one branch at a time [22]. The difference between App-EBC and Ran-EBC is greater for metric weights as oppose to angular weights. In particular the result shows Ang-Ran-EBC achieves the highest R2 for both case confirming previous finding that angular distances is an important factor describing aggregate pedestrian movement [2]. It also suggests that on aggregate, pedestrians may not be taking the optimal angular path in reaching the destination as long as it is preserving linearity and heading towards it [9].

Besides its potential, there are a number of limitations to the research. The research uses a simple empirical method in correlating aggregate movement behaviour and centrality indices. The improvement in goodness of fit is significant and consistent for both cases. However it is unclear if these differences are related to the actual processes of path selections or to the case study selections or to other unobserved missing factor. Thus, these results can serve as evidence for further research to consider a more exploratory approach in calculating centrality measures for pedestrian analysis. More empirical research especially at the disaggregated level and the neurological level is needed to understand the processes of the results. The second limitation is the research uses two simple exploratory path when calculating EBC. Future research shall consider other wayfinding strategies such as a multi-weights strategy (angular-metric), the anchor-based strategy, the hierarchical strategy, the scenic path strategy and the local optimal strategy when calculating betweenness centrality measures in studying pedestrian movement. Future research can also considered the notion of memory where agents can have different amount of knowledge when traversing.

Despite these limitations, these findings imply graph centrality measures can be useful as a descriptive measure to explain pedestrian movement variations with minimal data requirement. This is useful in countries where data is less complete. These findings also imply the concept of exploratory path is worth examining and that the adoption of a global optimum path should not be assumed when calculating centrality measures in studying pedestrian movement. Confirmation of these results can be used in expanding the path selections in pedestrian route choice models which can help pedestrian movement predictions. The results can also be used in refining pedestrian navigation applications such as routes recommendation and in robotics on navigation in complex urban environments. Most importantly, the results can also potentially contribute to the planning of more pedestriancentric cities, precisely on how people move and on how cities can be design to respond to it.

\section{ACKNOWLEDGMENT}

The authors wish to thank the reviewer for his comments and useful insights on the final version of the text. This work was supported by The Alan Turing Institute under the EPSRC grant EP/N510129/1.

\section{REFERENCES}

[1] U.Brandes, "A faster algorithm for betweenness centrality," Journal of mathematical sociology, vol. 25, no. 1, pp. 163-177, 2001.

[2] B.Hillier and S.Iida, "Network and psychological effects in urban environment," COSIT 2005, vol. 1, no. 1, pp. 475-490, 2005.

[3] E.W.Dijkstra, "A note on two problems in connection with graphs," Numerische Mathematik, vol. 1, no. 1, pp. 217-269, 1959.

[4] M.Newman, "A measure of betweenness centrality based on random walks," Soc.Networks, vol. 27, pp. 39-54, 2005.

[5] R.G.Gollege, Wayfinding Behavior:Cognitive mapping and other spatial processes. Johns Hopkins University Press., 1999.

[6] K.Lynch, The image of the city. MIT Press., 1960.

[7] J.Weisman, "Evaluating architectural legibility: Way-finding in the built environment," Environment and Behaviour, vol. 13, no. 2, pp. 189-204, 1981.

[8] B.Hillier and J.Hanson, The Social Logic of Space. Cambridge University Press, 1984.

[9] C.R.Dalton, "The secret is to follow your nose: Route path selection and angularity," Environment Behaviour, vol. 35, no. 1, pp. 107-131, 2003.

[10] S.J.Zhu and D.Levinson, "Do people use the shortest path? an empirical test of wardrop's first principle," PLoS One, vol. 10, no. 8, 2015.

[11] M.J.Chadwick, A.E.J.Jolly, D.P.Amos, D.Hassabis, and H.J.Spiers, "A goal direction signal in the human entorhinal/subicular region," Current Biology, vol. 25, no. 1, pp. 87-92, 2015.

[12] J.O'Keefe and J.Dostrovsky, "The hippocampus as a spatial map: preliminary evidence fromm unit activity in the freely-moving rat," Brain Research, vol. 34, pp. 171-175, 1971.

[13] Y.Dabaghian, V.L.Brandt, and L.M.Frank, "Reconceiving the hippocampal map as a topological template," Elife, vol. 3, no. e03476, 2014

[14] A.H.Javadi, B.Emos, L.Howard, F.Zisch, Y.Yu, R.Knight, J.P.Silva, and H.J.Spiers, "Hippocampal and prefrontal processing of network topology to simulate the future," Nature Communications, vol. 8, no. 14652, 2017.

[15] H.Simon, "Bounded rationality and organisational learning," Organisational science, vol. 2, no. 1, pp. 125-134, 1991.

[16] E.Manley and T.Cheng, "Shortest path or anchor-based route choice: a large-scale empirical analysis of minicab routing london," Journal of Transport Geography, vol. 43, no. 1, pp. 123-139, 2015.

[17] J.Wiener and H.A.Mallot, "Fine-to-coarse route planning and navigation in regionalized environments," Spatial Cognition Computing, vol. 3, no. 4, pp. 331-358, 2003.

[18] S.Law, L.Versluis, and I.Kovalou, "A least angular local shortest path," Space Syntax Symposium 11 Proceedings, no. 0, p. TBC, Forthcoming.

[19] A.Bevelas, "A mathematical model for group structure," Human Organisation, vol. 7, no. 1, pp. 16-30, 1948.

[20] L.Freeman, "A set of measures of centrality based on betweenness," Sociometry, vol. 40, no. 1, pp. 35-41, 1977.

[21] A.Penn, B.Hillier, D.Banister, and J.Xu, "Configurational modelling of urban movement networks," Environment and Planning B Planning and Design, vol. 24, no. 1, pp. 59-84, 1998.

[22] Q.J.Huys, N.Eshel, E.ONions, L.Sheridan, P.Dayan, and J.P.Roiser, "Bonsai trees in your head: how the pavlovian system sculpts goaldirected choices by pruning decision trees," PLoS Computational Biology, vol. 8, no. 3, 2012.

[23] V.Gilsing, B.Nooteboom, W.Vanhaverbeke, G.Duysters, and A.V.D.Oord, "Network embeddedness and the exploration of novel technologies: technological distance, betweenness centrality and density," Research Policy, vol. 37, pp. 1717-1731, 2008. 